\title{
Pathogenic significance of Klebsiella oxytoca in acute respiratory tract infection
}

\author{
JOAN T POWER, MARGARET -A CALDER
}

From the Department of Respiratory Medicine and the Bacteriology Laboratory, City Hospital, Edinburgh

ABSTRACT A retrospective study of all Klebsiella isolations from patients admitted to hospital with acute respiratory tract infections over a 27 -month period was carried out. Ten of the Klebsiella isolations from sputum and one from a blood culture were identified as Klebsiella oxytoca. The clinical and radiological features of six patients are described. Four of these patients had lobar pneumonia, one bronchopneumonia, and one acute respiratory tract infection superimposed on cryptogenic fibrosing alveolitis. One of the patients with lobar pneumonia had a small-cell carcinoma of the bronchus. We concluded that Klebsiella oxytoca was of definite pathogenic significance in these six patients and of uncertain significance in the remaining five patients. Klebsiella oxytoca has not previously been described as a specific pathogen in the respiratory tract. Close co-operation between clinicians and microbiologists in the management of patients with respiratory infections associated with the Enterobacteriaceae is desirable.

Klebsiella oxytoca has not previously been described as a specific respiratory pathogen. Bacillus oxytoca was first isolated by Flugge from a specimen of sour milk in $1886 .{ }^{12}$ It was not until 1963 that the organism was accepted as a member of the genus Klebsiella and then only with reluctance on the part of some authorities. ${ }^{3}$ To define more clearly the role of Klebsiella oxytoca as a pathogen in the respiratory tract we carried out a retrospective study of all Klebsiella species isolated from sputum specimens and blood cultures of patients admitted to the respiratory and chest units of the City Hospital from June 1979 to August 1981.

\section{Methods}

A total of 4800 patients were admitted during the period under review. About 12000 specimens of sputum were collected for culture and sensitivity testing. A minority of patients had blood cultures.

A Gram stain was made from the most purulent part of the sputum. A sputum isolate of Klebsiella was reported only if the organism was found with many pus cells in the Gram film. A blood agar plate and a heated blood agar plate containing 10 units of bacitracin per $\mathrm{ml}$ were inoculated and incubated in $10 \%$ carbon dioxide for 18 hours. One McConkey

Address for reprint requests: Dr JT Power, Department of Respiratory Medicine, City Hospital, Edinburgh EH10 5SB. agar plate was inoculated and incubated for 18 hours. The API 20E system (Analytal Product Inc) was used to identify the biochemical reactions. The two biochemical reactions which differentiate Klebsiella oxytoca from the Klebsiella pneumoniae organism are its ability to liquify gelatin and its indole positivity.

The disc diffusion method was used to establish antibiotic sensitivities. The following drugs were tested: ampicillin, chloramphenicol, co-trimoxazole, gentamicin, and tetracycline. Only one of the Klebsiella oxytoca isolates was sensitive to ampicillin.

\section{Results}

There were 110 Klebsiella isolations from sputum and one from blood culture. Eleven of these organisms, including the one isolated from blood culture, were identified as Klebsiella oxytoca. This was isolated from 11 patients (eight men and three women). Their ages ranged from 46 to 80 years (mean 68). Four of the 11 patients had radiological evidence of lobar pneumonia and one of bronchopneumonia. One patient had an acute respiratory tract infection superimposed on cryptogenic fibrosing alveolitis and five had acute exacerbations of chronic bronchitis.

Ten patients fulfilled the Medical Research Council's criteria for chronic bronchitis ${ }^{4}$ and one patient had cryptogenic fibrosing alveolitis. One had dissemi- 
nated oat-cell carcinoma of the bronchus with severe leucopenia caused by chemotherapy. Two patients had undergone partial gastrectomy for peptic ulceration. One patient had maturity-onset diabetes mellitus and one had chronic renal failure. Eight of the patients smoked 15-40 cigarettes a day while the remaining three were non-smokers. One patient had an excessive alcohol intake. In three patients Klebsiella oxytoca appeared to play a part in the pathogenesis of a slowly resolving pneumonia.

Case 1 A 46-year-old woman with a history of chronic bronchitis presented with left-upper-lobe pneumonia. Type 3 pneumococcal capsular antigens were detected in both her sputum and her serum by immunoelectrophoresis. Despite treatment with a combination of ampicillin and benzylpenicillin there was no appreciable radiological improvement over her first two weeks in hospital, during which time she remained febrile. Over the first few days the upper lobe increased in volume and there was bulging of the interlobar fissure (fig 1). During the third week cavitation was noted in the area of consolidation (fig 2). Klebsiella oxytoca was subsequently isolated from her sputum. The organism was resistant to ampicillin and benzylpenicillin and her treatment was thus changed to an appropriate combination of gentamicin and chloramphenicol. There followed a gradual clinical and radiological improvement. Fibreoptic bronchoscopy was carried out before her discharge and the findings were normal. A chest radiograph taken three months later showed residual fibrosis and loss of volume of the left upper lobe.

Case 2 A 55-year-old woman with a history of chronic bronchitis also presented with consolidation of the left upper lobe. She had been started on ampicillin and cloxacillin on the day before admission. No pathogens were isolated from the initial sputum culture, although type 3 pneumococcal antigens were detected in both sputum and serum. She was treated with a combination of benzylpenicillin, ampicillin, and cloxacillin, with an initial satisfactory clinical and radiological response. On her 10th day in hospital her temperature rose to $38.5^{\circ} \mathrm{C}$. At this stage Klebsiella oxytoca was isolated from her sputum. As the organism was resistant to her antibiotic regimen she was given a course of cotrimoxazole. She made an uneventful recovery, although again there was residual fibrosis in the left upper lobe on a chest radiograph taken six months after discharge from hospital.

Case 3 A 69-year-old man with a history of chronic bronchitis presented with radiological evi-

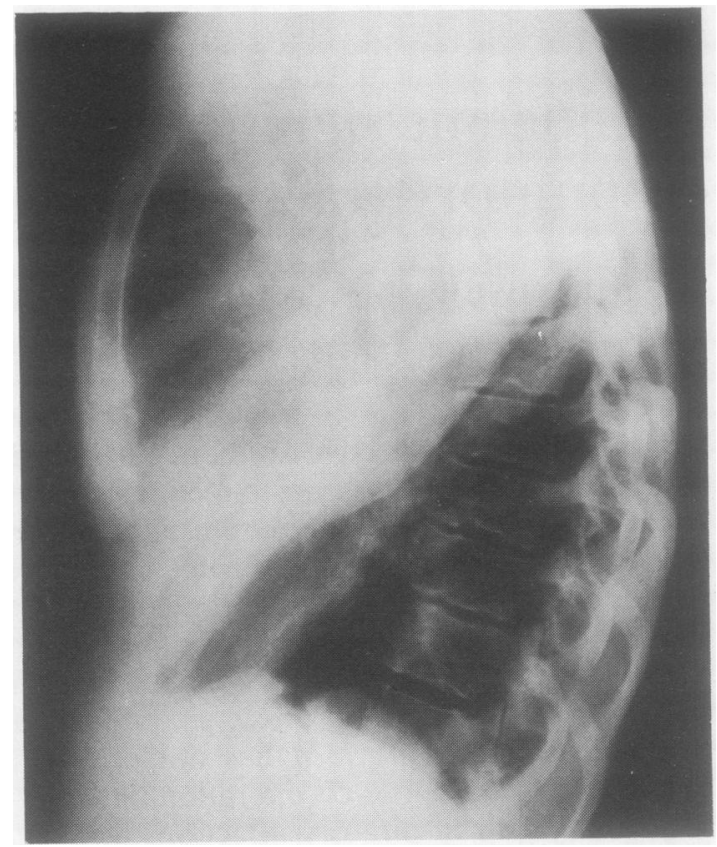

Fig 1 A left lateral chest radiograph of patient 1 two weeks after admission showing bulging of the interlobar fissure, evidence of an increase in volume of the left upper lobe.

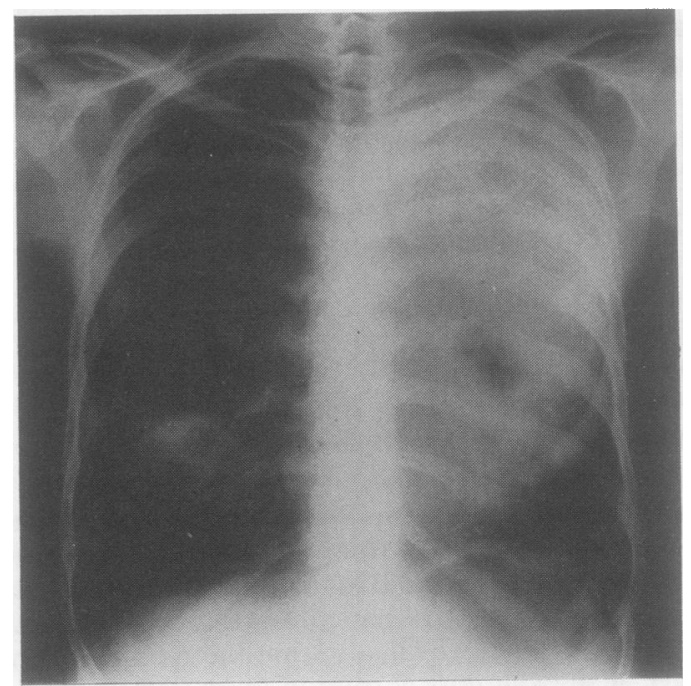

Fig 2 Chest radiograph of patient 1 two weeks after admission showing cavitation in the area of consolidation in the left upper lobe.

dence of right-upper-lobe pneumonia with abscess formation. Sputum culture on admission yielded aه profuse growth of Klebsiella oxytoca. He had not宂 previously received antibiotics. Treatment was a 


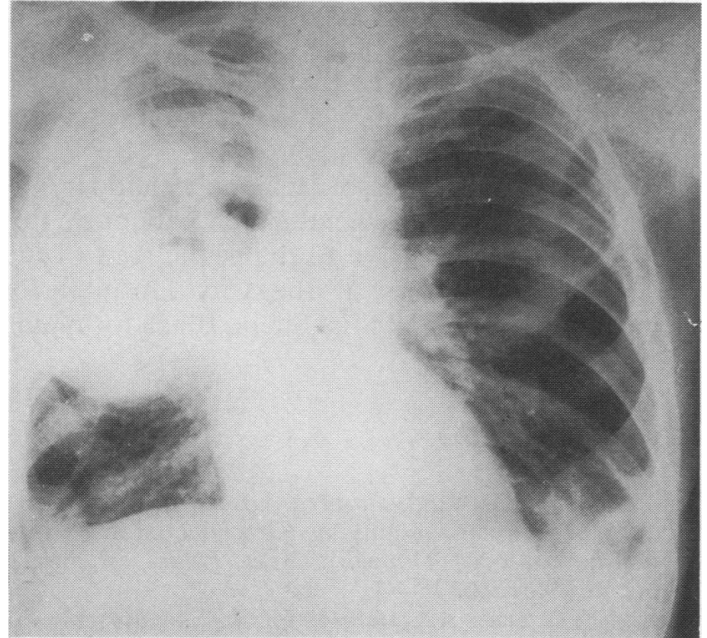

Fig 3 Chest radiograph of patient 3 on admission showing consolidation of the right upper lobe with abscess formation.

combination of ampicillin and co-trimoxazole; the organism was sensitive to both drugs. As there was no radiological improvement his antibiotic treatment was changed to a combination of benzylpenicillin, gentamicin, and metronidazole during his second week in hospital. His condition deteriorated, however, and he died two weeks later. There was no evidence of bronchial carcinoma at necropsy.

Case 4 A 61-year-old man with a small-cell carcinoma of the left-lower-lobe bronchus and distal left-lower-lobe consolidation had bacteraemia due to Klebsiella oxytoca. Eight days after his first course of cytotoxic chemotherapy he developed pyrexia. His total white cell count was $0.2 \times 10^{9} / 1$. Klebsiella oxytoca was isolated from a blood culture. A midstream specimen of urine was sterile on culture. Despite treatment with a combination of gentamicin, carbenicillin, and ampicillin he died the following day.

Case 5 A 72-year-old man with advanced cryptogenic fibrosing alveolitis and cor pulmonale was admitted in type 2 respiratory failure (as defined by $\mathrm{PaO}_{2}$ less than $8.0 \mathrm{kPa}(60 \mathrm{~mm} \mathrm{Hg})$ and a $\mathrm{PCO}_{2}$ above $\left.6.7 \mathrm{kPa}(50 \mathrm{~mm} \mathrm{Hg})^{5}\right)$. This was precipitated by an acute respiratory tract infection. He had taken ampicillin before admission. Klebsiella oxytoca was isolated from the initial sputum culture. He was not treated for this infection as he died before the results of sputum culture were available. The organism showed in vitro resistance to ampicillin. No postmortem examination was carried out.
Case 6 An 80-year-old man was admitted with a two-day history of confusion and of cough productive of brown sputum. A chest radiograph showed a right pleural effusion and patchy inflammatory infiltrates throughout both lung fields. He had not received antibiotics before admission and treatment was started with ampicillin. Klebsiella oxytoca was isolated from the initial sputum culture and so his antibiotic treatment was changed to gentamicin and co-trimoxazole. He made no appreciable response to treatment, however, and died on the fifth day after admission. There was no evidence of bronchial carcinoma at necropsy.

The remaining five patients presented with acute exacerbations of chronic bronchitis. They had no evidence of pneumonia on the chest radiograph. These patients had either been started on ampicillin before admission or responded to ampicillin, to which the organism showed in vitro resistance. It was therefore difficult to assess the pathogenicity of the Klebsiella isolations.

\section{Discussion}

Acute pneumonia caused by Klebsiella species is uncommon. In most previously reported studies it has accounted for only $1-2 \%$ of the organisms identified in cases of acute pneumonia. ${ }^{6-8}$ The exception was Tillotson's study, in which Klebsiella species accounted for $8 \%$ of the isolations; but his patients reflected the aged, debilitated population admitted to the medical wards of the Boston City Hospital. ${ }^{9}$

Klebsiella oxytoca appeared to play a significant part in producing a complicated lobar pneumonia in our first two patients. Both of these patients had a primary type 3 pneumococcal pneumonia. In the case of our first patient in particular, the course of the pneumonia resembled that of an acute klebsiella pneumonia rather than a pneumococcal pneumonia. Patient 3 had a pulmonary abscess in association with a Klebsiella oxytoca infection. His illness ran a relatively prolonged course. Klebsiella pneumonia can cause an indolent pulmonary abscess ${ }^{10}$ but this is a relatively uncommon manifestation of the infection. Likewise bronchopneumonia is unusual in association with acute klebsiella infection. ${ }^{11}$

Acute klebsiella pneumonia most frequently affects the upper lobes or the apical segments of the lower lobes. Three of our patients had an upperlobe pneumonia. There were other radiological features suggestive of acute klebsiella pneumonia in our first six patients. An increase in volume of the affected lobe with resultant bulging of the interlobar fissure and a tendency to abscess formation are fea- 
tures of acute klebsiella pneumonia which may aid in its differentiation from an acute pneumococcal pneumonia. ${ }^{12}{ }^{13}$ There is also a higher incidence of pleural effusion and empyema formation in acute klebsiella pneumonia. Patients 1 and 2 developed residual parenchymal fibrosis, seen on the follow-up chest radiograph-another feature associated with acute klebsiella pneumonia. ${ }^{13}$

Bacteraemia reportedly occurs in $25 \%$ of cases of acute klebsiella pneumonia. ${ }^{11}$ Klebsiella oxytoca was of undoubted pathogenic significance in patient 4 , in whom it was isolated from a blood culture. The organism was very unlikely to have been a contaminant as there was a pure growth on culture and there were compatible clinical features.

The high incidence of concurrent diseases in our patients is of interest. Chronic bronchopulmonary diseases, alcoholism, and to a lesser extent diabetes mellitus have long been recognised as predisposing patients to acute klebsiella pneumonia ${ }^{13}$ and it would appear that this is true of Klebsiella oxytoca infections.

The role of the Enterobacteriaceae may be difficult to interpret in a clinical context. Not every Gram-negative bacillus in the sputum is a pathogen. Some are commensals and some appear during or after chemotherapy. When these organisms are isolated from a culture a Gram film should also be studied and the two examinations assessed together. When many bacilli are seen in association with large numbers of pus cells the organism is more likely to be of pathogenic significance. The importance of the bacteriological findings must always be assessed in relation to the patient's clinical condition. Close co-operation between the laboratory and clinicians is essential. The presence of an organism that is uncommon in the respiratory tract poses an additional problem. We have concluded, however, on the basis of our observations in this study that Klebsiella oxytoca is a potentially significant pathogen in the respiratory tract. It was most likely the primary infecting agent in four patients and caused a significant secondary infection in two patients with pneumococcal lobar pneumonia. When Klebsiella oxytoca is isolated from a patient who is failing to respond to current treatment or whose condition is deteriorating it should be treated aggressively with appropriate antibiotics.

We are grateful to Professor DC Flenley and Dr NW Horne for permission to include their patients in this study. We would also like to thank the staff of the bacteriology laboratory at the City Hospital for technical assistance and Miss Sheila Black for typing the manuscript.

\section{References}

${ }^{1}$ Flugge C. Die Mikroorganismen. Leipzig, 1886.

${ }^{2}$ Lautrop H. Gelatin-liquifying Klebsiella strains (Bacterium oxytoca) (Flugge). Acta Pathol Microbiol Scand 1956;39:375-84.

${ }^{3}$ Cowan ST, Steel KJ. Manual for the identification of medical bacteria. 2nd ed. Cambridge: Cambridge University Press, 1974:111.

${ }^{4}$ Medical Research Council. Definition and classification of chronic bronchitis. Lancet 1965;i:775-9.

${ }^{5}$ Flenley DC. Respiratory medicine. London: Ballière Tindall, 1981:123.

- Bath JCL, Boissard GPB, Calder MA, Moffat MAJ. Pneumonia in hospital practice in Edinburgh 19601962. Br J Dis Chest 1964;58:1-16.

${ }^{7}$ Sullivan RJ jun. Dowdle WR, Marine WM, Hierholzer JC. Adult pneumonia in a general hospital. Arch Intern Med 1972;129:935-42.

${ }^{8}$ White RJ, Blainey AD, Joy Harrison K, Clarke SKR. Causes of pneumonia presenting to a district general hospital. Thorax 1981;36:566-70.

9 Tillotson JP, Finland M. Bacterial colonisation and clinical superinfection of the respiratory tract complicating antibiotic treatment of pneumonia. J Infect Dis 1969;119:597-624.

${ }^{10}$ Reed WP. Indolent pulmonary abscess associated with Klebsiella and Enterobacter. Am Rev Respir Dis 1973;107:1055-9.

"Pierce AK, Sanford JP. Aerobic Gram negative bacillary pneumonias. Am Rev Respir Dis 1974;110:647.

12 Ritvo M, Martin F. The clinical and roentgen manifestations of pneumonia due to Bacillus mucosus cap- $\bigcirc$ sulatus (primary Friedlanders pneumonia). Am J Roentgenol 1949;62:211.

${ }^{13}$ Fraser FG, Paré JAP. Diagnosis of diseases of the chest. 2nd ed. Philadelphia: WB Saunders, 1978:ii,713-4. 